\title{
Efecto Cicatrizante de la Miel de Ulmo (Eucryphia cordifolia) Suplementada con Ácido Ascórbico como Tratamiento en Quemaduras
}

\author{
Healing Effect of Ulmo Honey (Eucryphia cordifolia) \\ Supplemented with Ascorbic Acid as Treatment in Burns
}

\author{
Carolina Schencke*; Adriana Vasconcellos ${ }^{* *}$; Jessica Salvo**** Carlos Veuthey ${ }^{* * * *}$ \& Mariano del Sol,***
}

SCHENCKE, C.; VASCONCELLOS, A.; SALVO, J.; VEUTHEY, C. \& DEL SOL, M. Efecto cicatrizante de la miel de Ulmo (Eucryphia cordifolia) suplementada con ácido ascórbico como tratamiento en quemaduras. Int. J. Morphol., 33(1):137-143, 2015.

RESUMEN: Debido al daño de la piel producto de quemaduras, los microorganismos encuentran condiciones favorables para su desarrollo, produciéndose desnaturalización, necrosis, exudado, congestión y edema. La miel posee actividad antioxidante, antiinflamatoria y antibacteriana. A su vez, el ácido ascórbico mejora la barrera epidérmica y reduce la contracción de heridas, entre otros. El objetivo de esta investigación fue evaluar morfológicamente la cicatrización de heridas con un tratamiento en base a miel de ulmo (Eucryphia cordifolia) suplementada con ácido ascórbico, comparando su efecto con miel de ulmo no suplementada, en heridas causadas por quemaduras de tipo B. La investigación fue aprobada por el Comité Ético Científico de la Universidad de La Frontera. Se trabajó con 15 cobayos (Cavia porcellus) distribuidos en tres grupos: MS (miel suplementada), M (miel), y Control+ (Hidrogel-Tull). Se provocó una quemadura en seco en el dorso del tórax de $1 \mathrm{~cm}$ de diámetro, que abarcó hasta el límite de la fascia profunda. Al día 10 de tratamiento, se extrajeron las biopsias y se depositaron en PBS. Posteriormente, fueron fijadas en formalina tamponada y procesadas para fines histológicos, realizándose las técnicas de VGE, VGC, PAS, y Sirius Red. El cobayo se utilizó como modelo animal por tener un metabolismo dependiente de ácido ascórbico. El grupo MS mostró un rápido debridamiento en comparación a los grupos control, con cierre epidermal en el 60\% de las muestras, observándose una fase proliferativa avanzada. El grupo M no regeneró la capa epidérmica y presentó una fase proliferativa inicial. Los resultados de este estudio evidenciaron que al suplementar la miel de ulmo con ácido ascórbico se logra una cicatrización más rápida, con respecto a la miel de ulmo no suplementada, en heridas causadas por quemaduras.

PALABRAS CLAVE: Cicatrización; Miel ulmo; Ácido ascórbico; Quemaduras.

\section{INTRODUCCIÓN}

El tratamiento de heridas por quemadura es un problema importante de la medicina clínica, donde los microorganismos encuentran condiciones extremadamente favorables para su desarrollo. El daño a la piel, una barrera protectora natural, conduce a la desnaturalización de la proteína, necrosis y exudado, donde predomina la congestión y edema. Como consecuencia de ello, los mecanismos de defensa, tanto humoral como celular, se deterioran. Por otro lado, la agresión térmica genera daño oxidativo, con una mayor actividad de radicales libres, lo que resulta en peroxidación lipídica, donde la correcta evaluación de la profundidad de la quemadura determina la decisión sobre el tratamiento, ya sea conservadora o quirúrgica local, (Gutiérrez, 2002). Muchas son las propiedades que se encuentran en api-terapéuticos, productos recogidos ytratados por abejas. La miel, además de generar una disminución de la inflamación, y promover la granulación, genera un control de radicales libres debido a su efecto antioxidante, limitando el daño y disfunción multiorgánica posterior, (Jull et al., 2008). Varios estudios han mostrado tasas significativamente reducidas de infección de heridas causadas por quemaduras, lo que indica que la miel esteriliza y promueve la granulación temprana. Aunque cada componente de la miel tiene su acción específica, la actividad

* Doctorado en Ciencias Morfológicas, Universidad de La Frontera, Temuco, Chile.

** Facultad de Medicina, Universidad de La Frontera, Temuco, Chile.

**** Facultad de Medicina, Universidad Mayor, Temuco, Chile.

Proyecto DIUFRO DI13-0044

CONICYT-PCHA/Doctorado Nacional/2014-21141130 
antimicrobiana in vivo puede ocurrir debido a una reacción sinérgica entre ellos (Seckam \& Cooper, 2013; Molan, 2009; Gethin et al., 2008).

Durante la cicatrización se destacan tres fases: inflamatoria, proliferativa y de remodelación, las que no son excluyentes entre sí, (Ramírez, 2010). En la fase proliferativa, el coágulo de fibrina, es reemplazado por tejido de granulación rico en colágeno III. Esto se debe a que en las primeras etapas del proceso de cicatrización los fibroblastos lo producen en forma activa, siendo reemplazado por colágeno I para restaurar la composición normal de la dermis. Algunos autores han evidenciado que la miel podría potenciar la producción de fibras colágenas a nivel dérmico durante la cicatrización de heridas. Ozlem Nisbet et al. (2010) observaron que la miel aceleró la síntesis de colágeno, lo que podría resultar en un aumento de la resistencia a la tracción de la fuerza tensil. Por otra parte, las pruebas histopatológicos realizadas por Jastrzes bska-Stojko et al. (2013), evidenciaron que el proceso de curación y de formación de la cicatriz en heridas causadas por quemaduras tratadas con miel con propóleo fue más rápida, en comparación con el control, aumentando el nivel de hidroxiprolina y colágeno en forma significativa.

En el caso de una lesión, el estrés asociado resulta en una mayor necesidad de vitamina $\mathrm{C}$, siendo requerida para la síntesis de colágeno y angiogénesis (Kamer et al., 2010), tema ampliamente estudiado en cobayo (Gould \& Woessner, 1956; Ross \& Bendit, 1961; Abercrombie et al., 1956; Robertson \& Schwartz, 1953). Por otro lado, las heridas por quemadura pueden tener un efecto oxidativo que requiera de un tratamiento que actúe en ese aspecto. Mucho se ha estudiado sobre el efecto de las terapias antioxidantes como tratamiento en quemados, donde la vitamina C logra eliminar los radicales libres en el espacio extracelular, mientras la vitamina E limpia eficazmente los radicales libres dentro de las células donde se producen metabolitos reactivos. Además, la vitamina $\mathrm{C}$ puede eliminar los radicales libres que se unen a la vitamina $\mathrm{E}$, logrando regenerar esta última. Horton (2003) estudió los efectos de una terapia basada en la administración de vitaminas post-quemadura, demostrando su acción en la síntesis de cardiomiocitos y secreción de citoquinas pro-inflamatorias. Horton indicó que el régimen utilizado, que incluía una combinación de antioxidantes (vitaminas C, E, y A) podría garantizar una adecuada acción de apoyo al efecto cicatrizante.

Dada la complejidad del tratamiento de quemaduras y la necesidad de estudios experimentales que proporcionen una mejor comprensión de los mecanismos implicados en la cicatrización de heridas, este estudio evaluó morfológicamente la cicatrización, con un tratamiento en base a miel de ulmo suplementada con ácido ascórbico y comparó su efecto con miel de ulmo no suplementada, en heridas causadas por quemaduras de tipo B.

\section{MATERIAL Y MÉTODO}

Estudio descriptivo experimental. Se utilizaron 15 cobayos (Cavia porcellus) adultos, sanos, con un peso aproximado de $450 \mathrm{~g}$ promedio, mantenidos con pellet $\mathrm{y}$ verdura ad libitum en el Centro de Excelencia en Estudios Morfológicos y Quirúrgicos (CEMyQ) de la Universidad de La Frontera, Temuco, Chile. Los animales fueron divididos en 3 grupos de 5 ejemplares: MVC (miel suplementada), M (miel) y Control+ (Hidrogel/Tull). La miel de ulmo fue analizada en su pureza (90\%) y esterilizada por rayos gamma. La injurias, de $1 \mathrm{~cm}$ de diámetro, se provocaron en el dorso de los cobayos, por contacto con calor seco, durante 3 segundos, abarcando hasta el límite de la fascia profunda. Se trabajó bajo efectos anestésicos intraperitoneales con una triada anestésica en base a atropina $40 \mathrm{mg} / \mathrm{kg}$, ketamina $5 \mathrm{mg} / \mathrm{g}$ y de xilazina $0,05 \mathrm{mg} / \mathrm{kg}$. Las curaciones se realizaron aplicando suero fisiológico tibio por jeringoterapia a $10 \mathrm{~cm}$ de distancia de la lesión, colocando una gasa impregnada con miel de ulmo suplementada con ácido ascórbico en el grupo MVC; miel de ulmo no suplementada en el grupo M; e Hidrogel/ Tull en el grupo Control+. Se realizó escarotomía en cada lesión para facilitar la impregnación de los tratamientos hasta el desprendimiento del tejido necrótico. Con este procedimiento se trataron y evaluaron diariamente las heridas hasta la obtención de las biopsias al día 10 postinjuria. La evaluación diaria se realizó con el Protocolo de Supervisión Diaria de Animales, Guía de Aspectos Bioéticos de la Experimentación Animal, CONICYT, 2009.

Las biopsias fueron lavadas en solución salina, depositadas en formalina tamponada al $10 \%$, deshidratadas e incluidas en paraplast (Histotec-Merck ${ }^{\circledR}$ ). Se realizaron cortes seriados de $10 \mu \mathrm{m}$ hasta llegar a la injuria, zona cortada en seriados de 4 um de espesor. Para el análisis histológico las secciones fueron teñidas con HE, Van Guiesson Colágena (VGC), Van Guiesson Elástica (VGE). Para el análisis histoquímico se utilizó ácido peryódico de Schiff (PAS) y Sirius Red. Las láminas fueron observadas en un microscopio óptico Motic BA410 y fotografiadas con cámara Moticam 580. Las imágenes fueron proyectadas en un monitor de pantalla plana marca LG®. El presente estudio es parte del proyecto DIUFRO DI13-0044, y fue aceptado por el Comité de Ética de la Universidad de La Frontera, Temuco, Chile. 


\section{RESULTADOS}

Se extrajeron biopsias de piel sana de distintos animales para evaluar normalidad. Las biopsias presentaron epidermis delgada, con gruesa capa córnea. La dermis papilar superficial estaba constituida por tejido conectivo laxo donde se observaron folículos pilosos y glándulas sebáceas y la dermis profunda por abundantes haces de colágeno distribuidos al azar. La biopsia del animal de prueba, quemado en el lomo por 3 segundos, evidenció daño profundo de la piel que comprometió hasta la hipodermis (quemadura tipo B).

Grupo MVC (miel suplementada). Al momento de la injuria se observó una escara necrótica inmediata por quemadura, la cual hace su desprendimiento espontáneo el día 6 post injuria, quedando expuesta una herida de $5+1 \mathrm{~mm}$ de diámetro, no sangrante, con presencia tejido granulatorio. Su diámetro se redujo $50 \%$ aproximadamente al día 10 post-injuria. Las biopsias presentaron una fase proliferativa avanzada. La epidermis se regeneró en un $60 \%$ de las biopsias. Éstas no presentaron estrato córneo. El resto de las capas se encontraban diferenciadas: estrato basal bien desarrollado, espinoso diferenciado y esbozo de estrato granular. La tinción PAS tuvo una leve reacción, evidenciando apenas la lámina basal. En la dermis cicatrizal se observó reacción fibroblástica con abundante proliferación de fibras colágenas, ausencia de folículos pilosos y glándulas sebáceas, a nivel superficial se observaron numerosos vasos sanguíneos y abundantes capilares pequeños (Fig. 1A). Esta zona presentó tejido conectivo denso, con delgadas fibras colágenas. Con la tinción de Van Giesson Colágena se logró diferenciar claramente la dermis superficial de la profunda. La dermis profunda presentó gruesas fibras colágenas dispuestas en haces (Fig. 2A). No se observaron fibras elásticas en la dermis cicatrizal; sin embargo, éstas fueron abundantes en los márgenes de la herida (Fig. 3A). Con la tinción de Sirius Red se distinguieron fibras colágenas verdes en la dermis cicatrizal, poco intensas, muy finas, rodeadas de fibras colágenas color rojo, el cual se tornó más intenso hacia la hipodermis (Fig. 4A).
Grupo M (miel no suplementada). El tejido necrótico generado por la quemadura se desprendió espontáneamente entre los días 6-7 post-injuria, quedando expuesta una herida de $4+$ $1 \mathrm{~mm}$ de diámetro, no sangrante, con tejido granulatorio sin evidencia macroscópica de epitelización. Su diámetro se redujo 50\% aproximadamente, al día 10 post injuria. Las biopsias presentaron una fase proliferativa inicial al día 10 post injuria. No hubo regeneración epidermal. Se observó neoformación de vasos sanguíneos en toda la dermis cicatrizal, mayoritariamente en dermis superficial, con abundante celularidad y fibroblastos activos (Fig. 1B). La dermis cicatrizal se encontró en plena formación, se logró diferenciar de la hipodermis gracias a la tinción de Van Giesson Colágena (Fig. 2B). No se observaron fibras elásticas en dermis cicatrizal; sin embargo, éstas fueron abundantes en los márgenes de la herida, en dermis superficial (Fig. 3B). Con la tinción de Sirius Red se observaron finas fibras colágenas colorverde, rodeadas de fibras color rojo en dermis cicatrizal superficial, color que se tornó más intenso en la dermis profunda (Fig. 4B).

Grupo Control+(Hidrogel/Tull). El tejido necrótico generado por la quemadura se desprendió espontáneamente entre los días 8-9 post injuria, quedando expuesta una herida cruenta de aspecto granulatorio, sangrante al contacto, sin evidencia macroscópica de epitelización. Presentó un diámetro de $6+1$ $\mathrm{mm}$, con una reducción del 15\% al día 10 de tratamiento. Las biopsias presentaron una fase proliferativa avanzada con reacción fibroblástica al día 10 post injuria. La epidermis se regeneró en un $40 \%$ de las biopsias, encontrándose en estado de diferenciación. Se observó neoformación de vasos sanguíneos en la dermis superficial (Fig. 1C). En la zona cicatrizal se observó un tejido conectivo abundante en fibras colágenas, que se encontraban regularmente dispuestas y diferenciadas de la hipodermis, donde las fibras colágenas formaron gruesos haces (Fig. 2C). No se observaron fibras elásticas en la dermis cicatrizal; sin embargo, éstas fueron abundantes en los márgenes de la herida, en dermis superficial (Fig. 3C). Con la tinción de Sirius Red se observó la dermis cicatrizal con fibras colágenas color verde, y fibras colágenas color rojo intenso a nivel de la dermis profunda (Fig. 4).
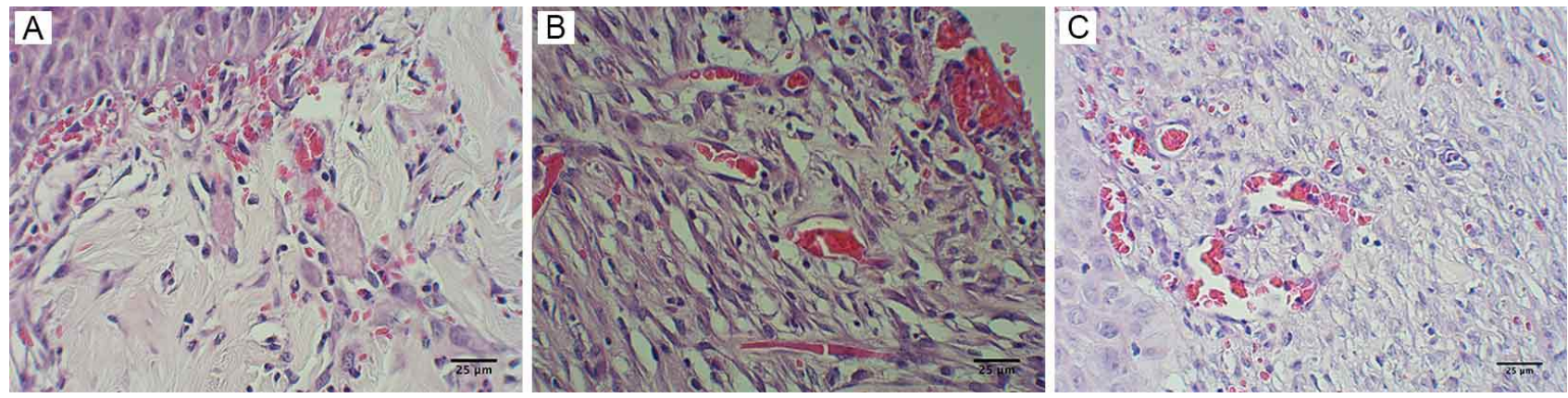

Fig. 1. Tinción HE para cada grupo en estudio. A. Biopsia del grupo MVC. Dermis cicatrizal con numerosos vasos sanguíneos. Abundan pequeños capilares. B. Grupo M. Se observa neoformación de vasos sanguíneos en toda la dermis cicatrizal, mayoritariamente en la dermis superficial, donde aún no hay desarrollo epidérmico. C. Grupo Control+. Neoformación de vasos sanguíneos sólo en dermis superficial. 

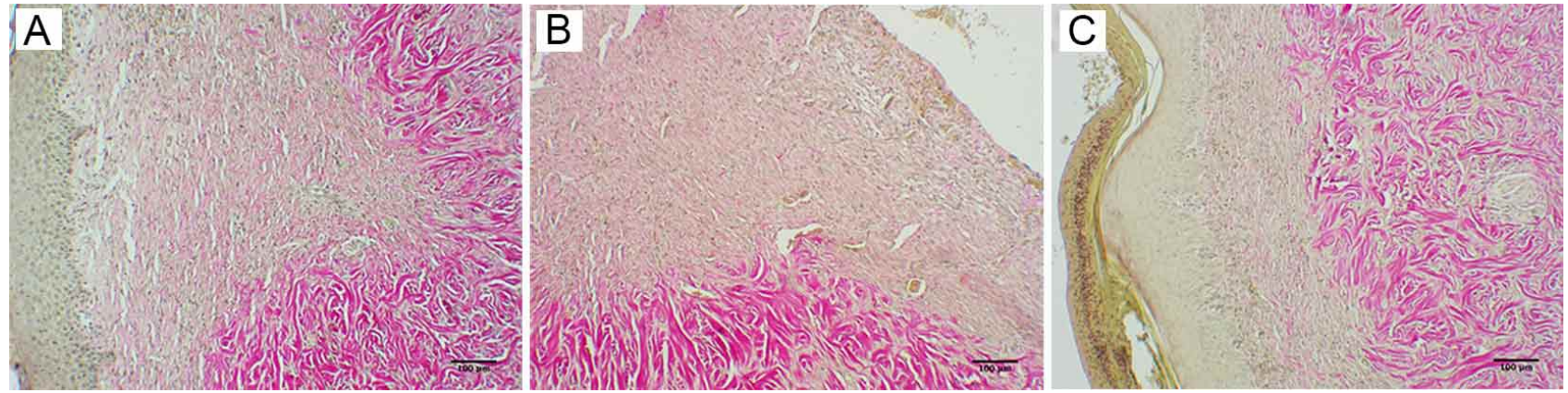

Fig. 2. Tinción VGC para cada grupo en estudio. A. Biopsia del grupo MVC. Presenta cierre epidermal. En dermis se observa el área cicatrizal con tejido conectivo denso, rico en fibras colágenas, diferenciado de la hipodermis que presenta gruesas fibras colágenas dispuestas en haces. B. Biopsia del grupo M. No presenta epidermis, la dermis cicatrizal se encuentra en plena formación, bien diferenciada de la dermis profunda. C. Biopsia del grupo Control+. Presenta epidermis, la dermis se observa en similares condiciones que el grupo MVC.
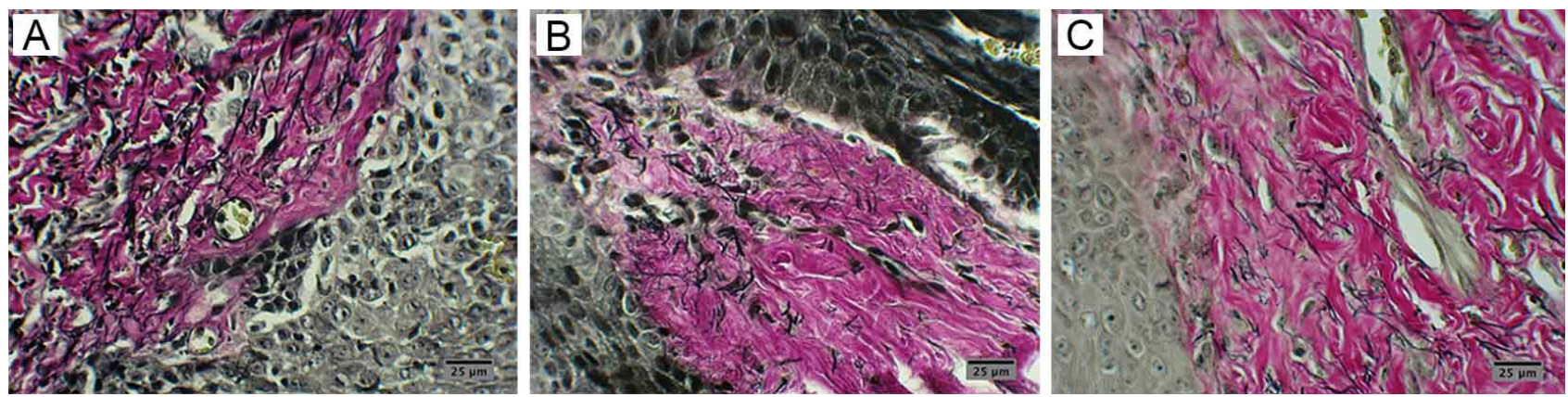

Fig. 3. Tinción VGE para cada grupo en estudio. A. Biopsia grupo MVC. A. Se observaron abundantes fibras elásticas en dermis cicatrizal, superficial, de los márgenes de la herida. B. Biopsia grupo M. Se observaron fibras elásticas en márgenes de la lesion. C. Biopsia grupo Control+. Presencia de fibras elásticas en márgenes de la lesion, las que son muy abundantes.

\section{DISCUSIÓN}

El cobayo (Cavia porcellus) se utilizó como modelo animal por tener un metabolismo dependiente del ácido ascórbico (Kramer et al., 1979) y estar indicado como animal de experimentación para evaluar lesiones por quemaduras (González, 2002; Sullivan et al., 2001). Según Kaufman et al. (1990) este animal mantiene una constante de espesor de piel ideal para estudios experimentales de cicatrización. Por otro lado, el estudio de heridas causadas por quemaduras es un excelente modelo para el análisis de los componentes principales del proceso de cicatrización: epitelización, contracción y formación de la cicatriz (Rodríguez-Bigas et al., 1988).

Establecer el origen de la miel permite darle un valor agregado, ya que muchos atributos y características dependen de las especies vegetales de proveniencia (Montenegro \& Fredes, 2008). La miel de ulmo puede ser una alternativa terapéutica para la cicatrización de heridas, ya que exhibe un efecto peróxido como poder antimicrobiano, siendo más efectiva que la miel de manuka en este aspecto (Sherlock et al., 2010).
Las condiciones básicas para la cicatrización del tejido dañado son: limpieza de la herida, activación de macrófagos, proliferación de fibroblastos, producción de matriz extracelular, y la formación de nuevos vasos sanguíneos (neoangiogénesis). El tratamiento de heridas debe tener una buena debridación para permitir la formación de tejido granular y la subsecuente epitelización. La acción debridante de la miel podría deberse a la activación de proteasas en el tejido a través del peróxido de hidrógeno. Los resultados de este estudio mostraron una excelente debridación de los tejidos dañados y tratados con miel suplementada versus miel no suplementada y control, reduciendo la formación de edema tisular y estimulando tejido granular sin signos de infección.

Según lo observado en este estudio, la miel suplementada con ácido ascórbico promueve la formación de tejido epitelial con cierre epidermal, producción de fibras colágenas y rápido proceso de cicatrización. En estudios previos se analizó la acción del ácido ascórbico administrado 

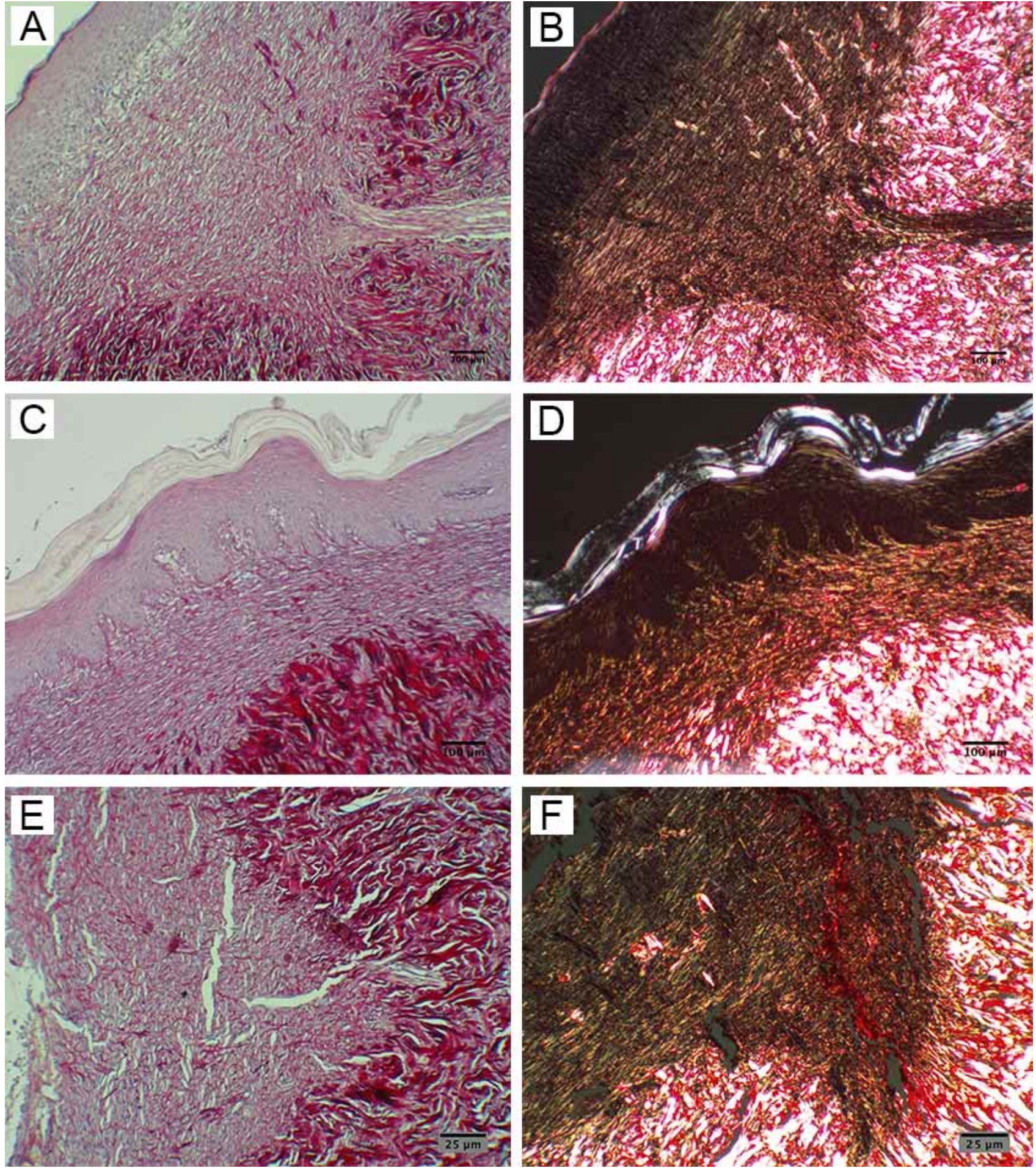

Fig. 4. Tinción Sirius Red para cada grupo en estudio. A1. Grupo MVC sin polarización. A2 Grupo MVC con polarización, se observa dermis cicatrizal con algunas fibras verdes, poco intensas, muy finas, rodeadas de fibras color rojo intenso. B2. Grupo M. Zona cicatrizal con fibras verdosas rodeado de fibras color rojo intenso. C2. Grupo Control+. Dermis cicatrizal confibras verdosas, dermis profunda con fibras color rojo intenso.

oralmente en la cicatrización de heridas en cobayo, lo que potenció la formación de fibras colágenas (Schencke et al.,
2013), evidenciando que las biopsias de los animales de prueba tratados sólo con suero fisiológico, no regeneraron la capa 
epidermal al día 10 post injuria. Se observaron tejido de granulación y edema, presentando una fase mixta con predominio agudo. Con el tratamiento propuesto, las biopsias se encontraron en un estado proliferativo avanzado al día 10 post-lesión siendo más rápida que en los grupos Miel y Control, concordando con los resultados de Kramer et al, quienes observaron una gran producción de colágeno cicatrizal en los cobayos que recibieron un suplemento oral de ácido ascórbico frente a los que no lo recibieron, pero tuvieron una dieta normal.

El colágeno cicatrizal se presentó como haces paralelos al tejido epitelial en las biopsias del grupo tratado con miel suplementada y controles, logrando una alta densidad. van Zuijlen et al. (2003), describieron el colágeno cicatrizal, el cual difiere significativamente de la piel normal. Los haces de colágeno se orientaron paralelos entre sí, organización que se volvió gradualmente más desordenada en las capas profundas, siendo esta forma de organización una característica de la cicatriz normotrotrófica, mientras que en la piel normal se observó una red tridimensional (Verhaegen, et al., 2009).

Barnes \& Morton, (1976) demostraron la presencia de colágeno tipo III en la cicatriz de cobayo, observando una mayor concentración de este colágeno en la dermis que rodeaba la cicatriz, gracias al análisis densitométrico de gelelectroforesis. Según Benavides (2008), este colágeno se secreta sólo en estadios tempranos de la reparación, apareciendo entre las 48 a 72 horas, siendo máximo entre los cinco a siete días. Después de un año o más, la dermis retorna gradualmente al fenotipo existente previo a la lesión, con predominio de colágeno tipo I. En el trabajo realizado, todos los grupos presentaron fibras colágenas tipo III en dermis cicatrizal, las que fueron observadas con la técnica de Sirius Red (Montes \& Junqueira, 1991).

Los resultados de este estudio estarían indicando que la miel de ulmo suplementada con ácido ascórbico potencia los efectos de la miel en la cicatrización de las heridas por quemaduras en epidermización y fase cicatrizacional. La miel proporciona un ambiente húmedo, bajo $\mathrm{pH}$, alta viscosidad, efecto higroscópico, y producción de peróxido de hidrógeno, estos factores juegan un papel combinado en la eficacia para el tratamiento de heridas por quemaduras. Los resultados descritos generan una línea de investigación en el tratamiento de heridas crónicas y quemaduras, donde la miel y el ácido ascórbico mostraron ser sustancias ideales como material de tratamiento, fácil de aplicar y remover.

SCHENCKE, C.; VASCONCELLOS, A.; SALVO, J.; VEUTHEY, C. \& DEL SOL, M. Healing effect of ulmo honey (Eucryphia cordifolia) supplemented with ascorbic acid as treatment in burns. Int. J. Morphol., 33(1):137-143, 2015.
SUMMARY: Skin damage from burns creates favorable conditions for microorganism development, producing denaturation, necrosis, discharge, congestion and edema. Honey contains antioxidant, anti-inflammatory and antibacterial properties. At the same time ascorbic acid reduces the epidermal barrier and wound contraction, among others. The objective of this research was to morphologically evaluate wound healing with treatment based on Ulmo honey (E. cordifolia) supplemented with ascorbic acid, comparing its effect with unsupplemented Ulmo honey, in wounds caused by type B burns. The research was approved by the Scientific Ethics Committee of the Universidad de La Frontera. We worked with 15 guinea pigs (Cavia porcellus) divided into three groups: SH (supplemented honey), H (honey), and Control + (HydrogelTulle). A $1 \mathrm{~cm}$ diameter dry burn was caused on the back of the chest and covered the extent of the deep fascia. At day 10 of treatment, biopsies were removed and placed in PBS. They were subsequently fixed in buffered formalin and processed for histological purposes, with VGE, VGC, PAS, and Sirius Red techniques. The guinea pig was used as an animal model for having a metabolism dependent on ascorbic acid. The SH group showed a rapid debridement compared to control groups, with epidermal closure in $60 \%$ of the samples, and advanced proliferative phase was observed. The $\mathrm{H}$ group did not regenerate epidermal layer and showed an initial proliferative phase. The results of this study showed that supplementing Ulmo honey with ascorbic acid achieves faster healing when compared to unsupplemented Ulmo honey in wounds caused by burns.

KEY WORDS: Healing; Ulmo honey; Ascorbic acid; Burns.

\section{REFERENCIAS BIBLOGRÁFICAS}

Abercrombie, M.; Flint, M. H.; \& James, D. W. Wound contraction in relation to collagen formation in scorbutic guinea-pigs. J. Embryol. Exp. Morphol., 4(2):167-75, 1956.

Barnes, M. J.; Morton, L. F.; Bennett, R. C.; Bailey, A. J. \& Sims, T. J. Presence of Type III Collagen in Guinea-Pig. Biochem. J., 157(1):263-6, 1976.

Benavides, J. Reparación de heridas cutáneas. Rev. Asoc. Col. Dermatol, 16(1):29-35, 2008.

Gethin, G. T.; Cowman, S. \& Conroy, R. M. The impact of manuka honey dressings on the surface $\mathrm{pH}$ of chronic wounds. Int. Wound J., 5(2):185-94, 2008.

Gould, B. S. \& Woessner, J. F. Biosyntesis of collagen. The influence of ascorbic acid on the proline, hidroxyproline, glycine, and collagen content of regenerating guinea pig skyn. J. Biol Chem., 226(1):289-300, 1956. 
González, R. Modelos experimentales para la evaluación de la acción cicatrizante de medicamentos. Rev. Cubana Farm., 36(3):189-96, 2002.

Gutiérrez, V. J. R. Daño oxidativo, radicales libres y antioxidantes. Rev. Cubana Med. Milit., 31(2):126-33, 2002.

Horton, J. W. Free radicals and lipid peroxidation mediated injury in burn trauma: The role of antioxidant therapy. Toxicology, 189(1-2):75-88, 2003.

Jastrzebska-Stojko, Z.; Stojko, R.; Rzepecka-Stojko, A.; KabalaDzik, A. \& Stojko, J. Biological activity of propolis-honey balm in the treatment of experimentally-evoked burn wounds. Molecules, 18(11):14397-413, 2013.

Jull, A. B.; Rodgers, A. \& Walker, N. Honey as a topical treatment for wounds. Cochrane Database Syst Rev., 2008. Vol. 4.

Kamer, E.; Unalp, H.; Gundogan, O.; Diniz, G.; Ortac, R.; Olukman, Murat; Derici, H. \& Onal, M. Effect of ascorbic acid on incisional wound healing in streptozotocin-induced diabetic rats. Wounds, 22(2):27-31, 2010.

Kaufman, T.; Lusthaus, S. N.; Sagher, U. \& Wexler, M. R.. Deep partial skin thickness burns: A reproducible animal model to study burn wound healing. Burns, 16(1):13-6, 1990.

Kramer, G. M.; Fillios, L. C. \& Bowler, E. Ascorbic acid treatment on early collagen production and wound healing in the guinea pig. J. Periodontol., 50(4):189-92, 1979.

Molan, P. Debridement of wounds with honey. J. Wound Technol., 5:12-7, 2009.

Montes, G. S. \& Junquiera, L. C. The use of the picrosiriuspolarization method for the study of the biopathology of collagen. Mem. Inst. Oswaldo Cruz, 86(Suppl. 3):1-11, 1991.

Montenegro, G. \& Fredes, C. Relación entre el origen floral y el perfil de elementos minerales en mieles chilenas. Gayana Bot., 65(1):122-5, 2008.

Ozlem Nisbet, H.; Nisbet, C.; Yarim, M.; Guler, A. \& Ozak, A. Original Research. Effects of Three Types of Honey on Cutaneous Wound Healing. Wounds, 22(11): 275-83, 2010.

Ramírez, G. Fisiología de la cicatrización cutánea. Rev. Fac. Salud, 2(2):69-78, 2010.

Robertson, W. B. \& Schwartz, B. Ascorbic acid and the formation of collagen. J. Biol. Chem., 201:689-96, 1953.

Rodríguez-Bigas, M.; Cruz, N. I. \& Suárez, A. Comparative evaluation of aloe vera in the management of burn wounds in guinea pigs. Plast. Reconstr. Surg., 81(3):386-9, 1988.

Ross, R. \& Benditt, E. P. Wound healing and collagen formation I.
Sequential changes in components of guinea pig skin wounds observed in the electron microscope. J. Biophys. Biochem. Cytol., 11:677-700, 1961.

Seckam, A. \& Cooper, R. Understanding how honey impacts on wounds: An update on recent research findings. Wounds Int., 4(1):20-4, 2013.

Sherlock, O.; Dolan, A.; Athman, R.; Power, A.; Gethin, G.; Cowman, S. \& Humphreys, H. Comparison of the antimicrobial activity of Ulmo honey from Chile and Manuka honey against methicillin-resistant Staphylococcus aureus, Escherichia coli and Pseudomonas aeruginosa. BMC Complement. Altern. Med., 10:47, 2010.

Schencke, C.; Salvo, J.; Vasconcellos, A. \& del Sol, M. Comparative Study of Healing in Burns Treatment Based on Ulmo Honey (Eucryphia cordifolia) and Oral Vitamin C in Guinea Pig (Cavia porcellus) versus Hydrogel. Int. J. Morphol., 31(3):839-44, 2013

Sullivan, T. P.; Eaglstein, W. H.; Davis, S. C. \& Mertz, P. The pig as a model for human wound healing. Wound repair and regeneration, 9(2): 66-76, 2001.

Verhaegen, P. D.; van Zuijlen, P. P.; Pennings, N. M.; van Marle, J.; Niessen, F. B.; van der Horst, C. M. \& Middelkoop, E. Differences in collagen architecture between keloid, hypertrophic scar, normotrophic scar, and normal skin: An objective histopathological analysis. Wound Repair Regen., 17(5):649-56, 2009.

Van Zuijlen, P. P.; Ruurda, J. J.; van Veen, H. A.; van Marle, J.; van Trier, A. J.; Groenevelt, F.; Kreis, R. W. \& Middelkoop, E. Collagen morphology in human skin and scar tissue: no adaptations in response to mechanical loading at joints. Burns, 29(5):423-31, 2003.

Dirección para correspondencia:

Carolina Schencke Figueroa

Doctorado en Ciencias Morfológicas

Facultad de Medicina

Universidad de La Frontera

Casilla $54 \mathrm{D}$

Temuco - CHILE

\section{Email: carolina.schencke@gmail.com}

Recibido: 09-11-2014

Aceptado: 22-12-2014 